\title{
Research on Cooperative Combat for Integrated Reconnaissance-Attack-BDA of Group LAVs
}

\author{
Li Bing, Li Jie, He Guanglin, and Li Dalin \\ School of Mechatronical Engineering, Beijing Institute of Technology, Beijing 100081, China \\ Correspondence should be addressed to He Guanglin; heguanglinbit@163.com
}

Received 31 August 2013; Revised 3 January 2014; Accepted 18 January 2014; Published 9 March 2014

Academic Editor: Vishal Bhatnagar

Copyright (C) 2014 Li Bing et al. This is an open access article distributed under the Creative Commons Attribution License, which permits unrestricted use, distribution, and reproduction in any medium, provided the original work is properly cited.

\begin{abstract}
LAVs (loitering air vehicles) are advanced weapon systems that can loiter autonomously over a target area, detect and acquire the targets, and then attack them. In this paper, by the theory of Itô stochastic differential, a group system was analyzed. The uniqueness and continuity of the solution of the system was discussed. Afterwards the model of the system based on the state transition was established with the finite state machine automatically. At last, a search algorithm was proposed for obtaining good feasible solutions for problems. And simulation results show that model and method are effective for dealing with cooperative combat of group LAVs.
\end{abstract}

\section{Introduction}

LAV is a new kind of aerial vehicle [1], which can loiter in the air over the targets. It comes out with the development of UAV and munitions. While a single LAV performing a single task will bring some benefits, greater benefits will come from the cooperation of group LAVs. The main motivation for group cooperation stems from the possible synergy, as the group performance is exceed the sum of the performance of the individual LAVs [2].

The recent development of group LAV technology has a great number of interests within the intelligence gathering and remote sensing communities [3]. Through cooperation, the team can reconfigure its distribution architecture to minimize the performance degradation to such expected failures. Such cooperation should take advantage of the following capabilities available to the group: Global Information, Resource Management, and Robustness.

In the past few years, the problem of cooperation problems has been widely studied by researchers. Group cooperation is the strongest degree of cohesive group action. Consider a set of LAVs that have been designated to be part of the group; the group could have more than one payoff function that it wishes to optimize, which would then entail multiobjective optimization [4].

However, the current study, whether for its cooperation with automatic organization or conscious cooperation, is carried out only for a specific problem. Flight formation is investigated in [5-7]. In [8], the method of Mixed Integer Linear Programming approach is applied to path planning. A similar cooperative path planning problem is also discussed in [9-11]. Cooperative task allocation problem is discussed in $[12,13]$ using game theory. In $[14,15]$, cooperative task allocation problem is discussed by intelligent algorithm. Collision and obstacle avoidance are discussed in [16, 17]. Cooperative information consensus is discussed in [18-20] and many others [21, 22].

In this paper we focus on autonomous LAVs, which are designed to operate as a pack of vehicles that search, detect, and attack targets. We develop analytic probability model for analyzing some design and operational aspects relating to group LAVs. The rest of the paper is organized as follows. We describe the differential equation model with finite state machine automatically. And the optimization function is established based on the combat efficiency. Finally, we provide some test results and give our conclusions.

\section{Problem Formulation and Analysis}

2.1. The Behaviors in the Process of Cooperative Combat. Each LAV has an identical set of behaviors governed by the same controller. A LAV can be in one of four possible situations: Searching, Attacking, BDA, and Removed.

The rules of the behavior are as in Algorithm 1. 
If the LAV satisfied the physical characteristics and communication capability, then over the target area searching for valuable targets;

If the LAV confirms the targets are the valuable, then acquires the target and attacks it;

If After the attacks on targets, then over the target area for BDA;

If achieve the targets' $\mathrm{BDA}$, then enter the next round of decisions;

Algorithm 1

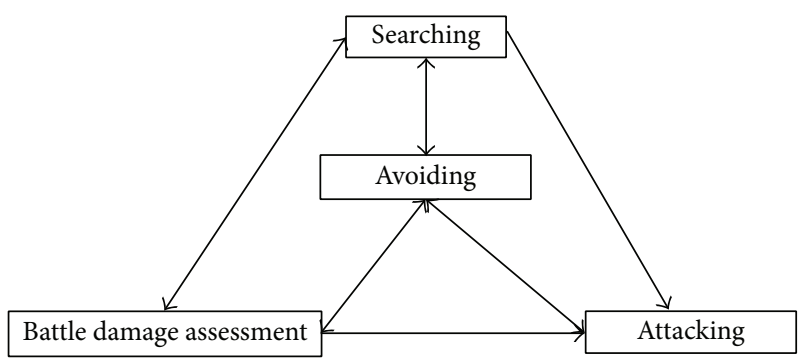

FIGURE 1: State diagram of the group LAVs behaviors.

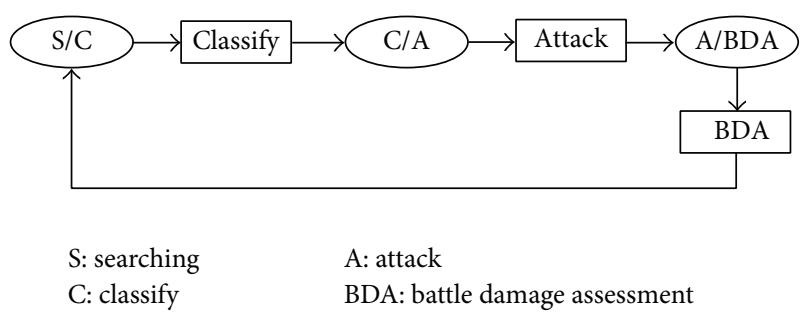

FIGURE 2: LAV mission timeline.

Figure 1 shows the sequence of behaviors that the LAV engages in during the combat process. Figure 2 describes the aforementioned mission time parameters.

2.2. Influence Analysis of the Group LAVs System. Consider dynamic model described by the following equation;

$$
\begin{aligned}
& F_{i}(t)=\left(\prod_{j=1}^{N} U_{j i}(t)^{\alpha_{j i}}\right) S_{i}(t)^{\beta_{i}} E_{i}(t)^{\gamma_{i}}, \\
& i=1,2, \ldots, N ; j=1,2, \ldots, N, j \neq i,
\end{aligned}
$$

where $U_{j i}(t)$ is the influence from $j$ individual of Swarms system, $S_{i}(t)$ is the UAV $i$ self-influence, $E_{i}$ is the positive factor of environment, and $\alpha_{i, j}$ and $\beta_{i}$ are the constant parameters.

In order to describe the influence function from the system science we use the Itô stochastic differential.

Set

$$
U_{i}(t)^{\alpha_{i}}=\prod_{j=1}^{N} U_{j i}(t)^{\alpha_{j i}},
$$

satisfying $\beta_{i}+\alpha_{i}=1, i=1, \ldots, N$.
According to the Itô stochastic differential we can get the stochastic differential equations of the factor function $F_{i}(t)$ :

$$
\begin{aligned}
d F_{i}(t)= & \frac{\partial F_{i}(t)}{\partial\left(U_{i}, S_{i}, E_{i}, t\right)}+\frac{1}{2} \frac{\partial^{2} F_{i}(t)}{\partial\left(U_{i}^{2}, E_{i}^{2}, S_{i}^{2}\right)} \\
& +\frac{\partial^{2} F_{i}(t)}{\partial U_{i} \partial S_{i}} d U_{i}(t) d S_{i}(t)+\frac{\partial^{2} F_{i}(t)}{\partial U_{i} \partial E_{i}} d U_{i} d E_{i} \\
& +\frac{\partial^{2} F_{i}(t)}{\partial S_{i} \partial E_{i}} d S_{i} d E_{i} .
\end{aligned}
$$

Theorem 1. Consider the following Itô stochastic differential equation:

$$
\begin{aligned}
d F_{i}(t)= & \frac{\partial F_{i}(t)}{\partial\left(U_{i}, S_{i}, E_{i}, t\right)}+\frac{1}{2} \frac{\partial^{2} F_{i}(t)}{\partial\left(U_{i}^{2}, E_{i}^{2}, S_{i}^{2}\right)} \\
& +\frac{\partial^{2} F_{i}(t)}{\partial U_{i} \partial S_{i}} d U_{i}(t) d S_{i}(t)+\frac{\partial^{2} F_{i}(t)}{\partial U_{i} \partial E_{i}} d U_{i} d E_{i} \\
& +\frac{\partial^{2} F_{i}(t)}{\partial S_{i} \partial E_{i}} d S_{i} d E_{i} .
\end{aligned}
$$

Structure a Borel measurable function and satisfied Lipschitz condition. Then if

$$
F_{i}(x, y, z, 0)=\text { Const }, \quad E\left(F_{i}(x, y, z, 0)\right)^{2}<+\infty,
$$

the differential equation $d F_{i}(t)$ has the unique and continuous solution $F_{i}(t)$ and convergence in probability.

Proof. The stochastic differential equation is derived by Variable replacement, which is described as follows:

$$
\begin{gathered}
F_{U_{i} U_{i}}=\alpha_{i}\left(\alpha_{i}-1\right) \rho^{2} U_{i}^{\alpha_{i}} S_{i}^{\beta_{i}} E_{i}^{\gamma_{i}} d t, \\
F_{S_{i} S_{i}}=\beta_{i}\left(\beta_{i}-1\right) \sigma^{2} U_{i}^{\alpha_{i}} S_{i}{ }^{\beta_{i}} E_{i}{ }^{\gamma_{i}} d t, \\
F_{E_{i} E_{i}}=\gamma_{i}\left(\gamma_{i}-1\right) \delta^{2} U_{i}^{\alpha_{i}} S_{i}{ }^{\beta_{i}} E_{i}^{\gamma_{i}} d t, \\
F_{U_{i} S_{i}}=\alpha_{i} \beta_{i} \sigma \rho U_{i}^{\alpha_{i}} S_{i}^{\beta_{i}} E_{i}{ }^{\gamma_{i}} d t, \\
F_{U_{i} E_{i}}=\alpha_{i} \gamma_{i} \rho \delta U_{i}^{\alpha_{i}} S_{i}^{\beta_{i}} E_{i}^{\gamma_{i}} d t, \\
F_{S_{i} E_{i}}=\alpha_{i} \gamma_{i} \sigma \delta U_{i}^{\alpha_{i}} S_{i}^{\beta_{i}} E_{i}^{\gamma_{i}} d t .
\end{gathered}
$$


Then the equation $d F_{i}(t)$ converts into the following form:

$$
\begin{aligned}
& d F_{i}(t)=\left(\left[\begin{array}{c}
\frac{1}{2}\left(\alpha_{i}\left(\alpha_{i}-1\right) \rho^{2}+\beta_{i}\left(\beta_{i}-1\right) \sigma^{2}+\gamma_{i}\left(\gamma_{i}-1\right) \delta^{2}\right) \\
\left(\alpha_{i} \rho+\beta_{i} \sigma+\gamma_{i} \delta\right)
\end{array}\right]\right. \\
& \left.+\left[\begin{array}{c}
\left(\alpha_{i} \beta_{i} \sigma \rho+\alpha_{i} \gamma_{i} \rho \delta+\beta_{i} \gamma_{i} \sigma \delta\right) \\
0
\end{array}\right]\right)^{T}\left[\begin{array}{l}
d t \\
d z
\end{array}\right] F_{i}(t) .
\end{aligned}
$$

So we structure the Borel measurable function as follows:

$$
\begin{aligned}
g\left(t, F_{i}\right)= & \frac{1}{2}\left(\left(\alpha_{i}\left(\alpha_{i}-1\right) \rho^{2}+\beta_{i}\left(\beta_{i}-1\right) \sigma^{2}+\gamma_{i}\left(\gamma_{i}-1\right) \delta^{2}\right)\right. \\
& \left.+2\left(\alpha_{i} \beta_{i} \sigma \rho+\alpha_{i} \gamma_{i} \rho \delta+\beta_{i} \gamma_{i} \sigma \delta+n \rho+s \alpha+\delta m\right)\right) \\
& \times F_{i}(t) h\left(t, F_{i}\right)=\left(\alpha_{i} \rho+\beta_{i} \sigma+\gamma_{i} \delta\right) F_{i}(t)
\end{aligned}
$$

According to the lemma in [23] lemma, we will prove that the Borel measurable function satisfied the Lipchitz condition:

$$
\begin{aligned}
& \left|g\left(t, F_{i 1}\right)-f\left(t, F_{i 2}\right)\right|+\left|h\left(t, F_{i 1}\right)-\delta\left(t, F_{i 2}\right)\right| \\
& =\left|\frac{1}{2}\left(\alpha_{i}\left(\alpha_{i}-1\right) \rho^{2}+\beta_{i}\left(\beta_{i}-1\right) \sigma^{2}+\gamma_{i}\left(\gamma_{i}-1\right) \delta^{2}\right) F_{i}(t)\right| \\
& \times\left|F_{i 1}-F_{i 2}\right|+\left|\alpha_{i} \beta_{i} \sigma \rho+\alpha_{i} \gamma_{i} \rho \delta+\beta_{i} \gamma_{i} \sigma \delta+n \rho+s \alpha+\delta m\right| \\
& \times F_{i}(t)\left|F_{i 1}-F_{i 2}\right|+\left(\alpha_{i} \rho+\sigma \beta_{i}+\gamma_{i} \delta\right)\left|F_{i 1}-F_{i 2}\right| \\
& \leq\left|\frac{1}{2}\left(\alpha_{i}\left(\alpha_{i}-1\right) \rho^{2}+\beta_{i}\left(\beta_{i}-1\right) \sigma^{2}+\gamma_{i}\left(\gamma_{i}-1\right) \delta^{2}\right)\right| \\
& \times\left|F_{i 1}-F_{i 2}\right|+\left|\left(\alpha_{i} \beta_{i} \sigma \rho+\alpha_{i} \gamma_{i} \rho \delta+\beta_{i} \gamma_{i} \sigma \delta\right)\right|\left|F_{i 1}-F_{i 2}\right| \\
& +\left|\left(n \rho+s \alpha_{i}+\delta m+\alpha_{i} \rho+\sigma \beta_{i}+\gamma_{i} \delta\right)\right|\left|F_{i 1}-F_{i 2}\right|, \\
& \left|g\left(t, F_{i}\right)\right|^{2}+\left|h\left(t, F_{i}\right)\right|^{2} \\
& =\frac{1}{4}\left(\alpha_{i}\left(\alpha_{i}-1\right) \rho^{2}+\beta_{i}\left(\beta_{i}-1\right) \sigma^{2}+\gamma_{i}\left(\gamma_{i}-1\right) \delta^{2}\right. \\
& \left.+2 \alpha_{i} \beta_{i} \sigma \rho+2 \alpha_{i} \gamma_{i} \rho \delta+2 \beta_{i} \gamma_{i} \sigma \delta+2 n \rho+2 s \alpha\right)^{2} F_{i}^{2} \\
& +\left(\alpha_{i} \rho+\sigma \beta_{i}+\gamma_{i} \delta\right)^{2} F_{i}^{2} \\
& \leq\left\{\left[\alpha_{i}\left(\alpha_{i}-1\right) \rho^{2}+\beta_{i}\left(\beta_{i}-1\right) \sigma^{2}+\gamma_{i}\left(\gamma_{i}-1\right) \delta^{2}\right.\right. \\
& \left.+\left(\alpha_{i} \beta_{i} \sigma \rho+\alpha_{i} \gamma_{i} \rho \delta+\beta_{i} \gamma_{i} \sigma \delta+n \rho+s \alpha+\delta m\right)\right]^{2} \\
& \left.+\left(\alpha_{i} \rho+\sigma \beta_{i}+\gamma_{i} \delta\right)^{2}\right\} F_{i}^{2} \\
& \omega_{1}=\left|\frac{1}{2}\left(\alpha_{i}\left(\alpha_{i}-1\right) \rho^{2}+\beta_{i}\left(\beta_{i}-1\right) \sigma^{2}+\gamma_{i}\left(\gamma_{i}-1\right) \delta^{2}\right)\right| \\
& +\left|\left(\alpha_{i} \beta_{i} \sigma \rho+\alpha_{i} \gamma_{i} \rho \delta+\beta_{i} \gamma_{i} \sigma \delta\right)\right| \\
& +\left|\left(n \rho+s \alpha_{i}+\delta m+\alpha_{i} \rho+\sigma \beta_{i}+\gamma_{i} \delta\right)\right|,
\end{aligned}
$$

$\omega_{2}=\sqrt{2} * \operatorname{Max}\left\{\left[\alpha_{i}\left(\alpha_{i}-1\right) \rho^{2}+\beta_{i}\left(\beta_{i}-1\right) \sigma^{2}+\gamma_{i}\left(\gamma_{i}-1\right) \delta^{2}\right.\right.$

$$
\begin{aligned}
& +\left(\alpha_{i} \beta_{i} \sigma \rho+\alpha_{i} \gamma_{i} \rho \delta+\beta_{i} \gamma_{i} \sigma \delta+n \rho\right. \\
& \left.\quad+s \alpha+\delta m)]^{2},\left(\alpha_{i} \rho+\sigma \beta_{i}+\gamma_{i} \delta\right)^{2}\right\},
\end{aligned}
$$

$\omega=\operatorname{Max}\left\{\omega_{1}, \omega_{2}\right\}$.

So we can get the equation and satisfy the Lipchitz condition as follows:

$$
\begin{gathered}
\left|g\left(t, F_{i 1}\right)-h\left(t, F_{i 2}\right)\right|+\left|\sigma\left(t, F_{i 1}\right)-\sigma\left(t, F_{i 2}\right)\right| \leq \omega\left|F_{i 1}-F_{i 2}\right| \\
\left|g\left(t, F_{i}\right)\right|^{2}+\left|h\left(t, F_{i}\right)\right|^{2} \leq \omega^{2}\left(1+\left|F_{i}\right|^{2}\right) .
\end{gathered}
$$

The conclusion that the influence function $F_{i}(t)$ has unique and continuous solution and convergence in probability 1 is correct.

\section{Mathematical Model and Analysis of System}

3.1. Mathematical Model of the System Based on the State Transition. Differential equation model was established using finite state machine automatically:

$$
\begin{aligned}
\frac{d N_{\text {Searching }}(t)}{d t}= & \frac{1}{\lambda_{\text {Avoiding }}} N_{\mathrm{AS}}(t)+\frac{1}{\lambda_{\mathrm{BDA}}} N_{\mathrm{BDA}}(t) \\
& -\alpha_{s} N_{\text {Searching }}(t) N_{\text {Tarket }}(t)-\alpha_{\mathrm{As}} N_{\text {Searching }}(t) \\
& \times\left(N_{\text {Searching }}(t)+N+B\right) \\
\frac{d N_{\mathrm{AS}}(t)}{d t}= & -\frac{1}{\lambda_{\text {Avoiding }}} N_{\mathrm{AS}}(t)+\alpha_{\mathrm{As}} N_{\text {Searching }}(t) \\
& \times\left(N_{\text {Searching }}(t)+N+B\right) \\
\frac{d N_{\mathrm{AA}}(t)}{d t}= & -\frac{1}{\lambda_{\text {Avoiding }}} N_{\mathrm{AA}}(t)+\alpha_{\mathrm{AA}} N_{\mathrm{Attacking}}(t) \\
& \times\left(N_{\text {Attacking }}(t)+N+B\right) \\
\frac{d N_{\mathrm{AB}}(t)}{d t}= & -\frac{1}{\lambda_{\text {Avoiding }}} N_{\mathrm{AB}}(t)+\alpha_{\mathrm{AB}} N_{\mathrm{BDA}}(t) \\
& \times\left(N_{\mathrm{BDA}}(t)+N+B\right) \\
\frac{d N_{\mathrm{Attacking}}}{d t} & (t) \\
\lambda_{\text {Attacking }} & \alpha_{s} N_{\mathrm{Searching}}(t) N_{\mathrm{Attacking}}(t) \\
&
\end{aligned}
$$




$$
\begin{aligned}
& \frac{d N_{\mathrm{BDA}}(t)}{d t}= \alpha_{\mathrm{AB}} N_{\mathrm{AB}}(t)-\frac{1}{\lambda_{\mathrm{BDA}}} N_{\mathrm{BDA}}(t)+\alpha_{\mathrm{AB}} N_{\mathrm{BDA}}(t) \\
& \times\left(N_{\mathrm{BDA}}(t)+N+B\right), \\
& \frac{d N_{\text {Tarket }}(t)}{d t}=-\alpha_{s} N_{\text {Searching }}(t) N_{\text {Tarket }}(t) .
\end{aligned}
$$

Parameters Notation. $N$ is the number of LAVs in the system, $N_{\text {Searching }}(t)$ is the number of LAVs in the searching state at time $t, N_{\text {Avoiding }}(t)$ is the number of LAVs in the avoiding state at time $t, N_{\text {Attacking }}(t)$ is the number of LAVs in the attacking state at time $t, N_{\mathrm{BDA}}(t)$ is the number of LAVs in the BDA state at time $t, N_{\text {Tarket }}(t)$ is the number of targets at time $t$, $\lambda_{\text {Avoiding }}, \lambda_{\text {Attacking, }}$, and $\lambda_{\mathrm{BDA}}$ are the average sustained time of Avoiding, Attacking, and $\mathrm{BDA}, \alpha_{S}$ is the rate of searching a valuable target, and $\alpha_{\mathrm{AS}}, \alpha_{\mathrm{AA}}, \alpha_{\mathrm{AB}}$ are the rate of encounter obstacles on Searching, Attacking, and BDA.

3.2. Cost Function Based on Effectiveness. In this paper we consider the total flight distance of the group system:

$$
\min \sum_{k=1}^{K}\left(\sum_{l=1}^{N_{c}} \sum_{i=1}^{N_{u}} \sum_{j=1}^{N_{\text {target }}} C_{l, i, j}^{X_{l-1}} x_{l, i, j}\right) .
$$

Parameter $x_{l, i, j} \in\{0,1\}$ is the decision variable and $s \in$ $(1,2,3)$ is the stages of the task; if $l \in s$, then $x_{l, i, j}=1$; else $x_{l, i, j}=0$, and $c_{l, i, j}^{x_{l-1}}$ is the distance for LAV $i$ to the target $j$.

Subject to

$$
\begin{gathered}
\sum_{l=1}^{N_{c}} \sum_{i=1}^{N_{u}} x_{l, i, j}=N, \quad j \in T, \\
\sum_{l=1}^{N_{c}} \sum_{j=1}^{N_{\text {target }}} r_{l, i, j}^{X_{l-1}} x_{l, i, j} \leq b_{i}, \quad i \in U, \\
d_{A \min } \geq d_{\text {min }}+v \Delta t_{1}, \\
d_{V \min } \geq d_{V \min }+v \Delta t_{2} .
\end{gathered}
$$

Parameter $b_{i}$ is the flight capability and $\Delta t_{m}$ is the min. time between the tasks.

3.3. Search Algorithm. According to the proposed cooperative combat, each LAV uses a cost function to select and update its behaviour. This method is quite flexible in that it allows the characterization of various mission-level objectives.

The flowchart depicting the optimization algorithm for the LAV group scenario is shown in Figure 3.

The data as in Tables 1 and 2.

In this example, eight LAVs are searching an area containing three targets of similar types.

LAVs trajectories through the 10 seconds are shown in Figure 4; at $t=10 \mathrm{~s}$ the targets are discovered by the searching

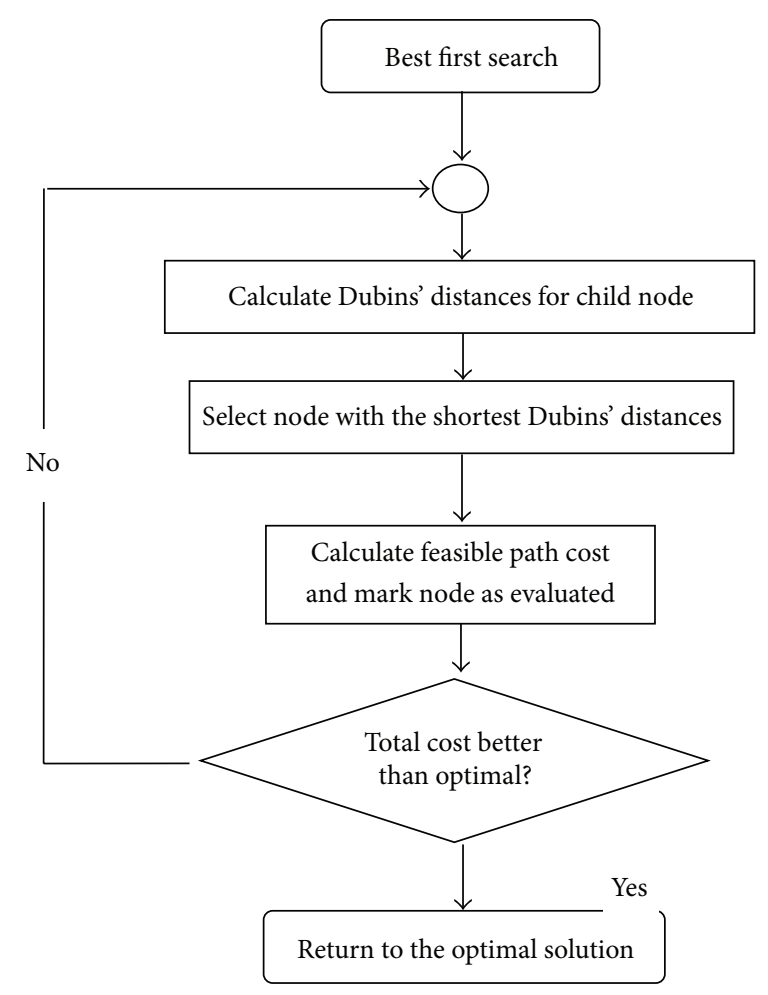

FIGURE 3: Search flowchart.

TABLE 1: Dynamics performance parameters.

\begin{tabular}{lc}
\hline Parameter & Value \\
\hline Loitering velocity & $100 \mathrm{~m} / \mathrm{s}$ \\
Maximum range & $180 \mathrm{~km}$ \\
Minimum turning radius & $500 \mathrm{~m}$ \\
\hline
\end{tabular}

TABLE 2: Initialization of the MAV.

\begin{tabular}{lccc}
\hline Index & Location $(\mathrm{km})$ & Orientation $(\mathrm{rad})$ & Period of flight time $(\mathrm{s})$ \\
\hline LAV1 & $(3.93,18.34)$ & 3.335 & 129 \\
LAV2 & $(5.02,5.72)$ & 4.895 & 635 \\
LAV3 & $(12.32,15.14)$ & 5.868 & 248 \\
LAV4 & $(9.47,15.07)$ & 0.816 & 422 \\
LAV5 & $(7.03,7.61)$ & 3.574 & 132 \\
LAV6 & $(16.62,11.36)$ & 2.949 & 481 \\
LAV7 & $(11.71,1.52)$ & 0.074 & 210 \\
LAV8 & $(10.99,1.08)$ & 2.118 & 523 \\
\hline
\end{tabular}

LAVs, nearly simultaneously. LAV1, 5 and 6 are assigned to verify the targets.

LAVs trajectories through the 50 seconds are shown in Figure 5 , at $t=39 \mathrm{~s}$ Target 3 is classified by LAV6, at $t=44 \mathrm{~s}$ Target 1 is classified by LAV1, at $t=49 \mathrm{~s}$ Target 2 is classified by LAV5, and then the LAVs perform attack on targets.

LAVs trajectories through the 115 seconds are shown in Figure 6, at $t=91 \mathrm{~s}$ Targets 1 was attacked by LAV4, then LAV2 performs BDA on target 1 .

LAVs trajectories through the 235 seconds are shown in Figure 7, at $t=120 \mathrm{~s}$ Targets 2 was attacked by LAV8, then 


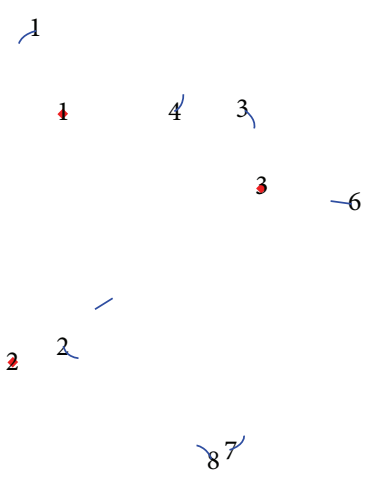

FIgURE 4: $T=10 \mathrm{~s}$ trajectories with respect to time.
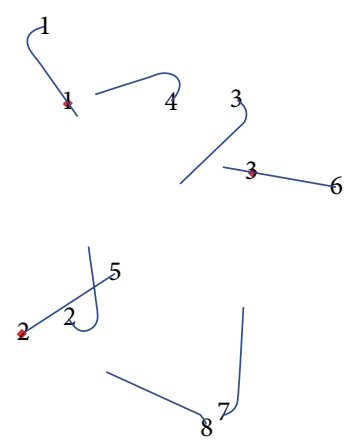

FIGURE 5: $T=50 \mathrm{~s}$ trajectories with respect to time.

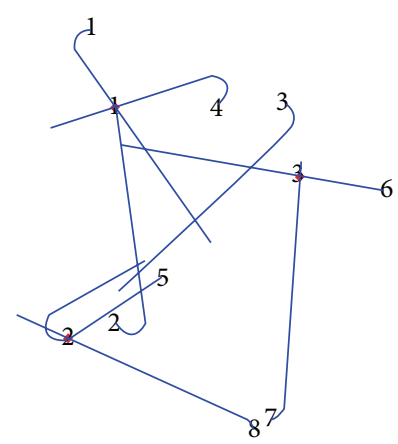

FIGURE 6: $T=115 \mathrm{~s}$ trajectories with respect to time.

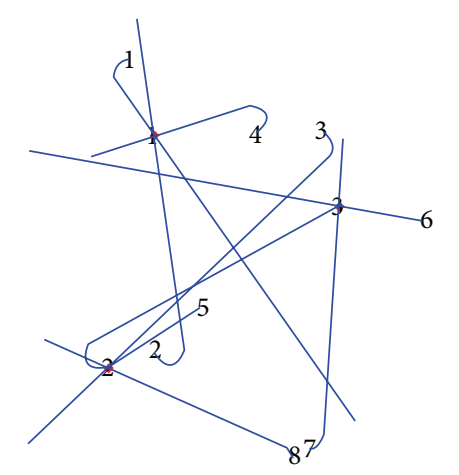

FIgURE 7: $T=235 \mathrm{~s}$ trajectories with respect to time.
LAV3 performs BDA on target 2, at $t=138 \mathrm{~s}$ Targets 3 was attacked by LAV7, then LAV5 performs BDA on target 3 .

At $T=230 \mathrm{~s}$, the tasks are completely finished by the group LAVs.

\section{Conclusion}

In this paper the problem of the cooperative combat associated with the group LAVs system has been solved. Afterwards, by the theory of Itô stochastic differential, a group system was analyzed. The model of the system based on the state transition was established with the finite state machine automatically. At last, a search algorithm was proposed for obtaining good feasible solutions for problems. And we took simulation tests to verify the conclusion.

\section{Conflict of Interests}

The authors declare that they have no conflict of interests regarding the publication of this paper.

\section{Acknowledgment}

This paper is supported by the National Defense Pre-Research Foundation of China (Grant no. B222011XXXX).

\section{References}

[1] D. Li, J. Li, Z. Guan, and J. Wang, "Improved GA based multi-LAVs' aerial delivery areas-choosing method," Applied Mechanics and Materials, vol. 157-158, pp. 1498-1505, 2012.

[2] T. Shima and S. Rasmussen, UAV Cooperative Decision and Control Challenges and Practical Approaches, Society for Industrial Mathematics, 2009.

[3] F. Namin, J. S. Petko, and D. H. Werner, "Analysis and design optimization of robust aperiodic Micro-UAV swarm-based antenna arrays," IEEE Transactions on Antennas and Propagation, vol. 60, no. 5, pp. 2295-2308, 2012.

[4] R. Luce and H. Raiffa, Games and Decisions: Introduction and Critical Survey, Dover Publications, New York, NY, USA, 1989.

[5] H. Kim and Y. Kim, "Trajectory optimization for unmanned aerial vehicle formation reconfiguration," Engineering Optimization, vol. 46, no. 1, pp. 84-106, 2014.

[6] I. Bayezit and B. Fidan, "Distributed cohesive motion control of flight vehicle formations," IEEE Transactions on Industrial Electronics, vol. 60, no. 12, pp. 5763-5722, 2013.

[7] Z. Chao, S. Zhou, L. Ming, and W. Zhang, "UAV formation flight based on nonlinear model predictive control," Mathematical Problems in Engineering, vol. 2012, Article ID 261367, 15 pages, 2012.

[8] J. Tisdale, Z. W. Kim, and J. K. Hedrick, "Autonomous UAV path planning and estimation: an online path planning framework for cooperative search and localization," IEEE Robotics and Automation Magazine, vol. 16, no. 2, pp. 35-42, 2009.

[9] A. T. Klesh, P. T. Kabamba, and A. R. Girard, "Path planning for cooperative time-optimal information collection," in Proceedings of the American Control Conference (ACC '08), pp. 19911996, June 2008. 
[10] Y. Kim, D. Gu, and I. Postlethwaite, "Real-time optimal mission scheduling and flight path selection," IEEE Transactions on Automatic Control, vol. 52, no. 6, pp. 1119-1123, 2007.

[11] P. B. Sujit and R. Beard, "Cooperative path planning for multiple UAVs exploring an unknown region," in Proceedings of the American Control Conference (ACC '07), pp. 5446-5451, July 2007.

[12] H. Chen, D. Shen, G. Chen, E. P. Blasch, and K. Pham, "Tracking evasive objects via a search allocation game," in Proceedings of the American Control Conference (ACC '10), pp. 6981-6986, July 2010.

[13] Q. Han, X. M. Wang, and J. Dang, "Research on the task allocation model and algorithm of MAS based on game theory," Computer Applications and Software, pp. 51-53, 2012.

[14] M. Darrah, E. Fuller, T. Munasinghe, K. Duling, M. Gautam, and M. Wathen, "Using genetic algorithms for tasking teams of raven UAVs," Journal of Intelligent and Robotic Systems, vol. 70, pp. 361-371, 2013.

[15] M. Yao, X. Wang, and M. Zhao, "Cooperative combat task assignment optimization design for unmanned aerial vehicles cluster," Journal of University of Electronic Science and Technology of China, pp. 723-727, 2013.

[16] R. Olfati-Saber and R. M. Murray, "Mesh stability of unmanned aerial vehicle cluster," in Proceedings of the American Control Conference (ACC'01), pp. 62-68, Arlington, Va, USA, June 2001.

[17] C. Tomlin, G. J. Pappas, and S. Sastry, "Conflict resolution for air traffic management: a study in multiagent hybrid systems," IEEE Transactions on Automatic Control, vol. 43, no. 4, pp. 509521, 1998.

[18] L. Bing, L. Jie, and H. KeWei, "Modeling and flocking consensus analysis for large-scale UAV swarms," Mathematical Problems in Engineering, vol. 2013, Article ID 368369, 9 pages, 2013.

[19] R. W. Beard and V. Stepanyan, "Information consensus in distributed multiple vehicle coordinated control," in Proceedings of the 43th IEEE Conference on Decision and Control, pp. 20292034, Maui, Hawaii, USA, December 2003.

[20] J. Luo, "Some new optimal control problem in UAV cooperative control with information flow constraints," in Proceedings of the American Control Conference (ACC'03), pp. 2181-2186, Denver, Colo, USA, June 2003.

[21] B. Li, "Stochastic process model of the multi-UAVs collaborative system based on state transition," in Proceedings of the 2012 International Conference on Modeling, Identification and Control, pp. 757-761, 2012.

[22] D. L. Li, Group decision making and control method for microunmanned aerial vehicle [Ph.D. thesis], Beijing Institute of Technology, 2011.

[23] B. Li, "Analysis of influence of multiple UAVs' coordination system," Journal of Information and Computational Science, vol. 10, no. 2, pp. 571-578, 2013. 


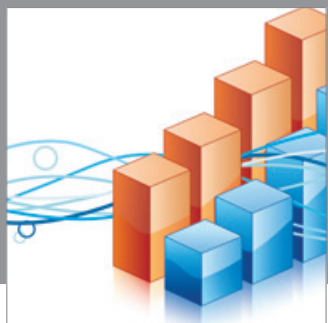

Advances in

Operations Research

mansans

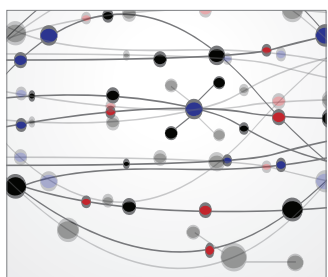

The Scientific World Journal
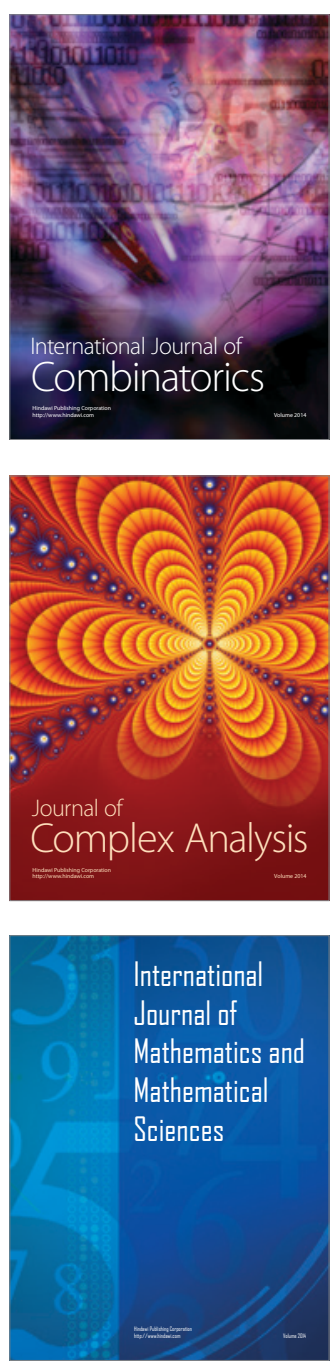
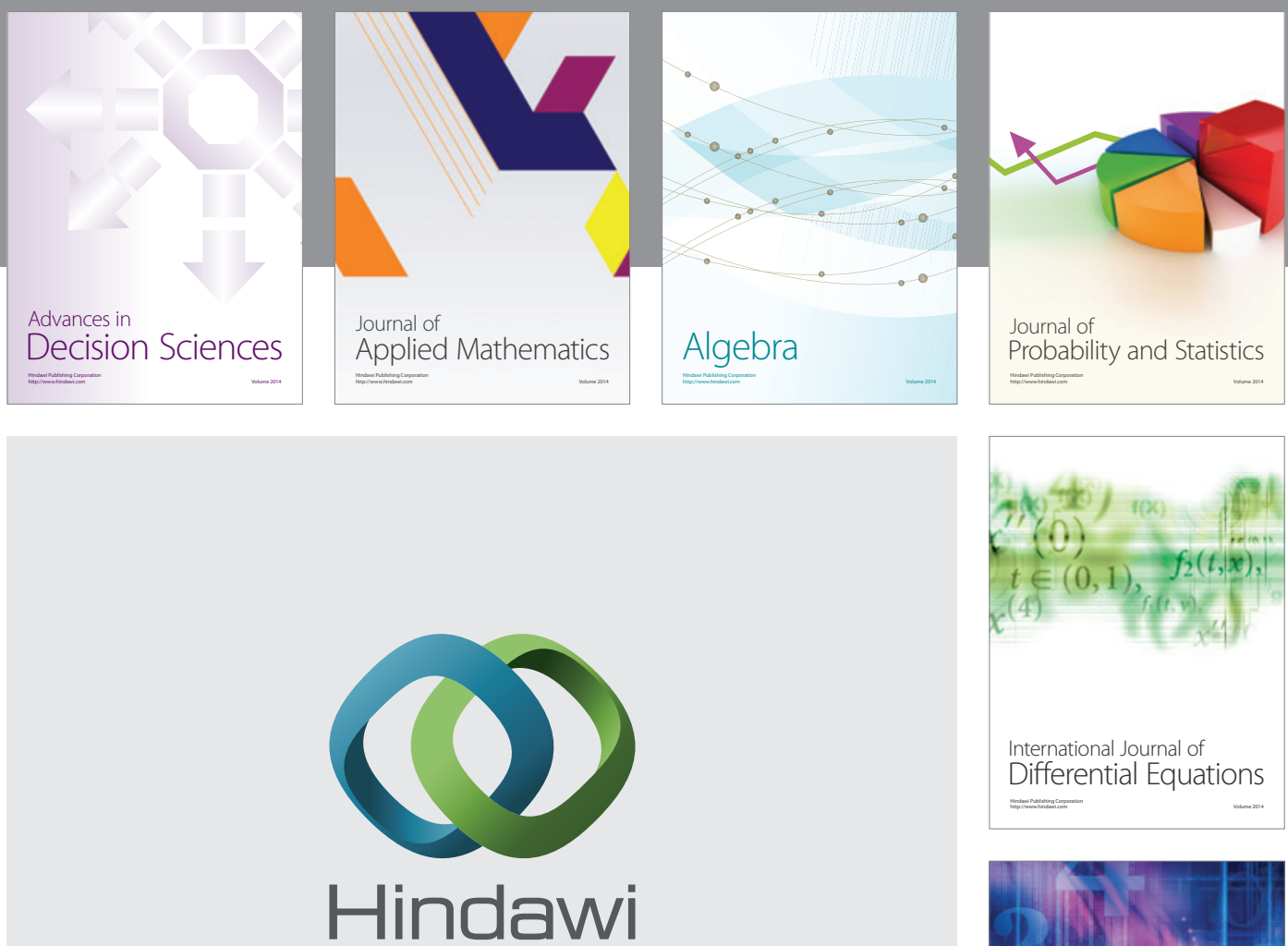

Submit your manuscripts at http://www.hindawi.com
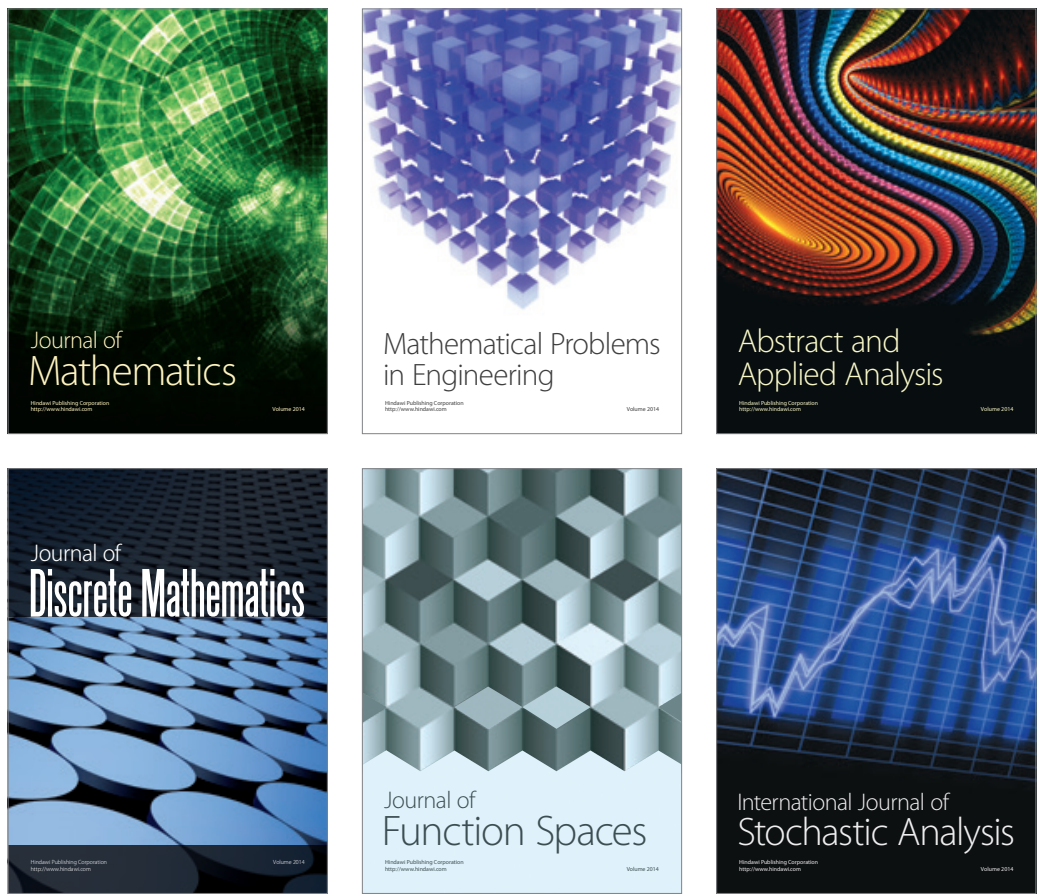

Journal of

Function Spaces

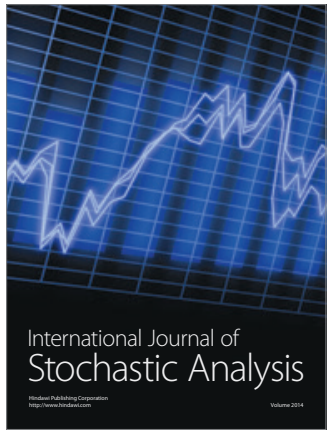

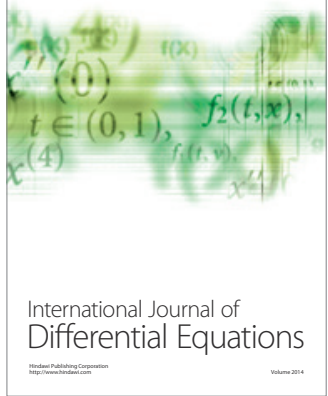
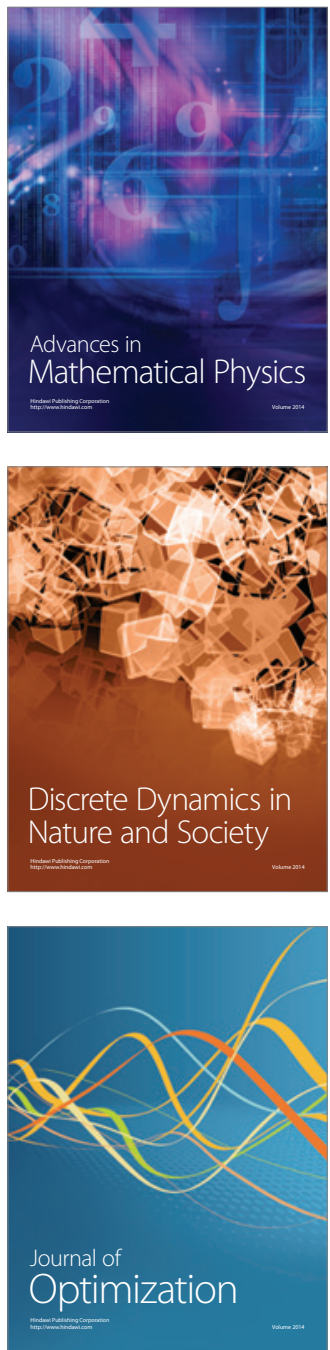